\title{
Telemedicine During the COVID-19 Pandemic: Moving from Physical to Virtual Outpatient Care
}

\author{
Jagdish P. Goyal ${ }^{1}\left[\right.$ D . Prawin Kumar ${ }^{1}$
}

Received: 23 July 2021 / Accepted: 30 July 2021 / Published online: 27 August 2021

(c) Dr. K C Chaudhuri Foundation 2021

The term telemedicine was first coined in the 1970s. It means healing at a distance [1]. There are more than 100 definitions of telemedicine have been identified. World health organization has also provided a broad definition of telemedicine [2]. These definitions highlight that telemedicine is an evolving science. It incorporates new advances in technology to improve patient outcomes.

The COVID-19 pandemic has forced to stop outpatient physical visits because of containment measures such as social distancing, lockdowns, and complete halt of public transport. It has become challenging to provide a continuum of care to patients, particularly of chronic diseases. Telemedicine has come as a rescue to overcome this problem. The Ministry of Health, Government of India, also issued guidelines because of the pandemic on telemedicine on March 20, 2020 [3].

Despite the fact and available guidelines, there were many challenges faced by both health care providers and patients. The recent study by Kumari et al. [4] provides insight into the role of telemedicine in the follow-up of children with chronic respiratory illnesses. It highlights the problem faced by both doctors and caregivers of the patients. This study also reported the satisfaction of caregivers with teleconsultation. The study concluded that follow-up care had been provided successfully with reasonable satisfaction of caregivers despite telemedicine being started hurriedly in the pandemic in most patients. The health care providers were also not adequately trained for telemedicine.

As the mobile and internet are readily available in the current era, it makes telemedicine more accessible. A systematic review and meta-analysis on the effects of telemedicine on asthma control and patients' quality of life reported that telemedicine could significantly improve asthma control compared with usual care [5]. The studies

Jagdish P. Goyal

jpgoyal@rediffmail.com

1 Department of Pediatrics, AIIMS, Jodhpur, Rajasthan 342001, India on chronic obstructive pulmonary disease (COPD) in adults also highlighted that patients accepted telemonitoring well [6]. It also reduced emergency hospital visits. Telemedicine is cost-effective since it eliminates transportation expenses.

Telemedicine may continue beyond the COVID-19 pandemic, especially in providing follow-up care to children with chronic diseases. We need to integrate telemedicine in medical education and provide adequate training to health care providers. Modern technology, including popular mobile applications with end-to-end encrypted facilities like WhatsApp, Telegram, Viber, etc., and smartphone devices may play a crucial role in telemedicine and also help to enhance patient satisfaction by video call. We need to standardize telemedicine care and devise validated online clinical examination tools to improve telemedicine practice and quality of care. Future efforts should also be made to make telemedicine more accessible to provide health care delivery to underserved, impoverished, and marginal children.

The future of telemedicine is very bright, and it may be a game-changer in current clinical practice. As we move ahead of virtual outpatient care, one must remember that few conditions still require a physical examination. Moreover, in-person visits provide more patient satisfaction.

To conclude, telemedicine might be an adequate substitute of face-to-face consultation. However, as the pandemic will evolve, we need to learn more about telemedicine use, patient expectations, and future application.

\section{Declarations}

Conflict of Interest None

\section{References}

1. Strehle EM, Shabde N. One hundred years of telemedicine: does this new technology have a place in paediatrics? Arch Dis Child. 2006;91:956-9. 
2. A health telematics policy in support of WHO's Health-for-all strategy for global health development : report of the WHO Group Consultation on Health Telematics, 11-16 December, Geneva. 1997. In: World Health Organization. 1998. Available at: https://apps.who.int/iris/handle/10665/63857. Accessed on 16 July 2021.

3. Telemedicine practice guidelines. In: mohfw. 2020. Available at: https://www.mohfw.gov.in/pdf/Telemedicine.pdf. Accessed on 16 July 2021.

4. Kumari J, Jat KR, Kabra SK. Role of telemedicine in followup care of children with respiratory illnesses at a tertiary care hospital - an ambispective observational study. Indian J Pediatr. 2021;4:1-5.
5. Chongmelaxme B, Lee S, Dhippayom T, Saokaew S, Chaiyakunapruk $\mathrm{N}$, Dilokthornsakul P. The effects of telemedicine on asthma control and patients' quality of life in adults: a systematic review and metaanalysis. J Allergy Clin Immunol Pract. 2019;7:199-216.

6. Jang S, Kim Y, Cho WK. A systematic review and meta-analysis of telemonitoring interventions on severe COPD exacerbations. Int J Environ Res Public Health. 2021;18:6757.

Publisher's Note Springer Nature remains neutral with regard to jurisdictional claims in published maps and institutional affiliations. 